\title{
Site-Specific Insertion Polymorphism of the MITE Alex-1 in the Genus Coffea Suggests Interspecific Gene Flow
}

\author{
Christine Dubreuil-Tranchant, ${ }^{1}$ Romain Guyot, ${ }^{1}$ Amira Guellim, ${ }^{1}$ Caroline Duret,,${ }^{1,2}$ \\ Marion de la Mare, ${ }^{1}$ Norosoa Razafinarivo, ${ }^{1,3}$ Valérie Poncet, ${ }^{1}$ Serge Hamon, ${ }^{1}$ Perla Hamon, ${ }^{1}$ \\ and Alexandre de Kochko ${ }^{1}$
}

\author{
${ }^{1}$ IRD, UMR DIADE, Centre IRD de Montpellier, BP 64501, 34394 Montpellier Cedex 5, France \\ ${ }^{2}$ UMR ECOFOG INRA, BP 709, 97387 Kourou Cedex, Guyane Française, France \\ ${ }^{3}$ FOFIFA, BP 1444, Ambatobe, Antananarivo 101, Madagascar
}

Correspondence should be addressed to Alexandre de Kochko, dekochko@ird.fr

Received 1 March 2011; Accepted 16 July 2011

Academic Editor: A. Rus Hoelzel

Copyright ( 12011 Christine Dubreuil-Tranchant et al. This is an open access article distributed under the Creative Commons Attribution License, which permits unrestricted use, distribution, and reproduction in any medium, provided the original work is properly cited.

\begin{abstract}
Miniature Inverted-repeat Transposable Elements (MITEs) are small nonautonomous class-II transposable elements distributed throughout eukaryotic genomes. We identified a novel family of MITEs (named Alex) in the Coffea canephora genome often associated with expressed sequences. The Alex-1 element is inserted in an intron of a gene at the CcEIN4 locus. Its mobility was demonstrated by sequencing the insertion site in C. canephora accessions and Coffea species. Analysis of the insertion polymorphism of Alex-1 at this locus in Coffea species and in C. canephora showed that there was no relationship between the geographical distribution of the species, their phylogenetic relationships, and insertion polymorphism. The intraspecific distribution of $C$. canephora revealed an original situation within the E diversity group. These results suggest possibly greater gene flow between species than previously thought. This MITE family will enable the study of the C. canephora genome evolution, phylogenetic relationships, and possible gene flows within the Coffea genus.
\end{abstract}

\section{Introduction}

Recently, a new type of molecular marker based on the insertion polymorphism of transposable elements (TEs) was shown to be particularly effective for plant diversity studies [1-4]. Because of their repeated nature and, in some cases, their great number $[5,6]$, these mobile genetic elements may be inserted at different loci in the genome where they lead to mutations or chromosomal rearrangements. Their activity is responsible for considerable natural polymorphism that can be used to study within and between species diversity and to identify possible population genetic structure and phylogenetic relationships $[7,8]$.

Among these mobile genetic elements, MITEs (Miniature Inverted-repeat Transposable Elements) form a particular group. MITEs are short $(<600 \mathrm{bp})$ nonautonomous type II transposable elements that are often quite widely distributed in eukaryote genomes but at the same time are highly conserved, within a genome, in size and sequence, indicating that they might originate from a limited number of progenitors [9]. Their even distribution throughout plant genomes makes them an ideal tool for the study of genome evolution and genetic relationships [10-12].

Such elements could help to solve problematic phylogenetic relationships among species including those in the genus Coffea, which comprises 103 species originating from Africa, Madagascar, and several islands in the Indian Ocean [13]. A phylogenetic tree was constructed based on four plastid sequences: trnL intron, trnL-F IGS, rpl16, and accD-psal IGS and one nuclear repeated sequence: rDNA ITS [13]. The tree contains valuable information but also many un-solved relationships concerning the evolution of the genus and the speciation process, especially in Madagascar. Several approaches using TE could be used to solve this problem such as SSAP [14] and REMAP [15]. 


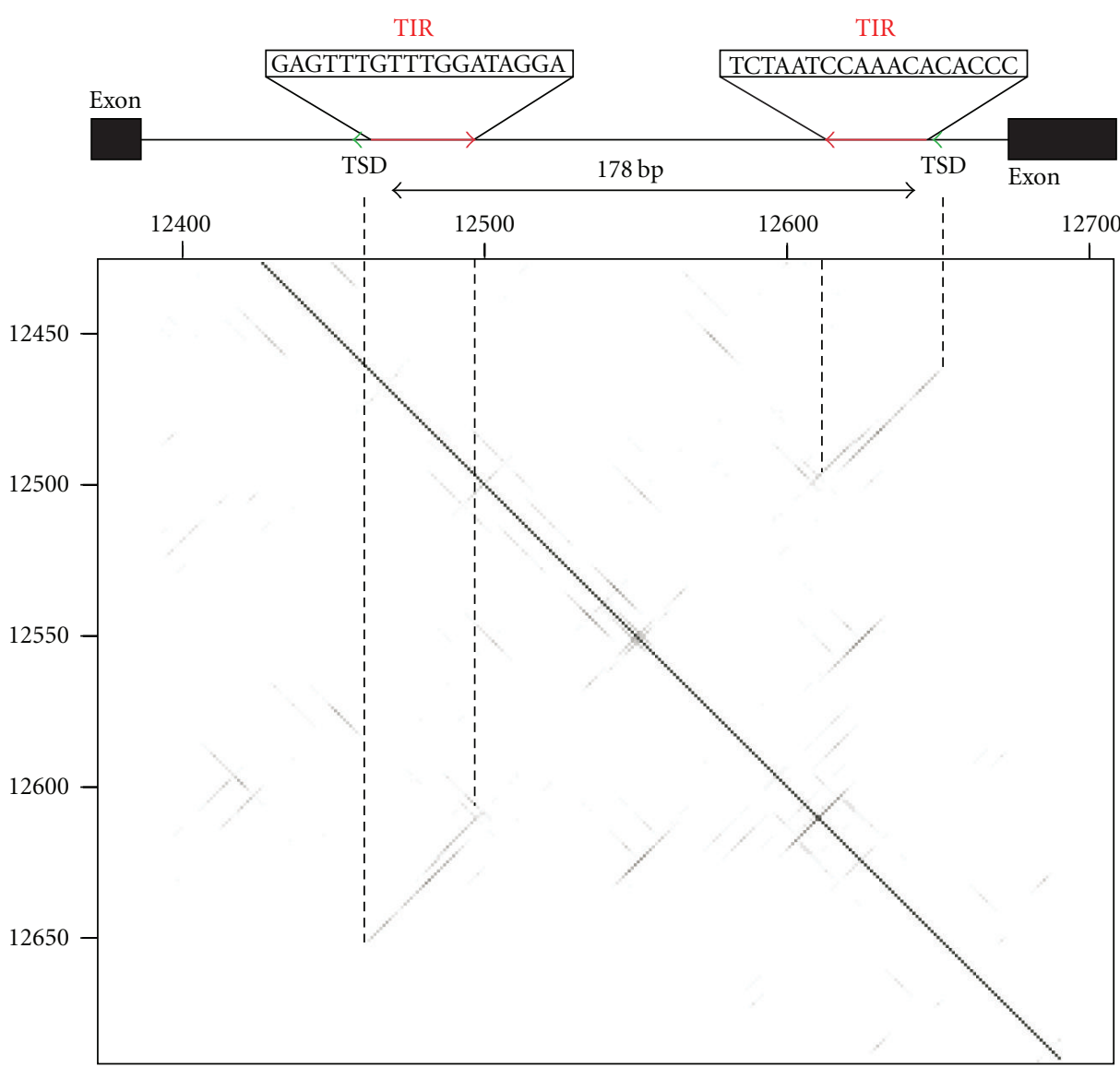

(a)

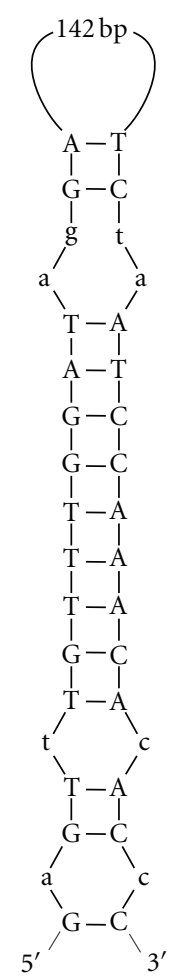

(b)

FIgURE 1: Structural characterization of the Alex-1 MITE. (a) Dot plot of the MITE against itself allowed the identification of the 18 bp terminal inverted repeats (in red). (b) Folding of Alex-1 revealed the typical stem loop structure of MITE elements.

Both approaches enable estimation of the genome-wide TE distribution. A third experimental procedure reveals sitespecific insertion polymorphism [16]. This method requires the identification of information on flanking sequences to facilitate the design of primers to detect polymorphism by PCR. Sequencing of a C. canephora BAC clone (46C02, accession no. EU164537) enabled identification of a new MITE, named Alex-1 (Figure 1), in the 12th intron of a gene (g3) of this BAC clone [17].

In this paper, we characterize this novel MITE family in the C. canephora genome. We also report the results of a study on the insertion polymorphism of the MITE Alex1 at the $g 3$ locus using PCR approaches on a representative set of Coffea species and a representative set of C. canephora diversity groups [18-21]. Our results revealed high insertion polymorphism of the Alex MITE at the $g 3$ locus, which was not linked to the phylogenetic relationships of the Coffea species studied here. Taken together, these results suggest greater gene flow between species than previously thought.

\section{Material and Methods}

2.1. Plant Material. Twenty-eight Coffea species grown in tropical greenhouses at the IRD center in Montpellier
(France) were used in this study. They represent the natural ecogeographical distribution of the genus. One plant from a related genus, Psilanthus, was included in the survey. One to 11 plants (genotypes) per species were analyzed (depending on the number of samples available in the collection) except for C. canephora for which 71 accessions were included (see Supporting Material available at doi: $10.4061 / 2011 / 3584122)$. For the latter species, DNAs from 12 plants from the Ugandan diversity group were kindly provided by P. Musoli from NARO/COREC (Uganda) and T. Leroy from CIRAD (France).

2.2. DNA Isolation. DNA was extracted and purified using Qiagen DNeasy mini Kits (Hilden, Germany) according to the manufacturer's instructions. DNA quantification was performed on a NanoDrop TM 1000 Spectrophotometer (LabTech, France).

2.3. Identification of the MITE Alex. De novo identification of the MITE was performed using dot-plot alignments (DOTTER software, [22]) based on the presence of inverted repeats at both ends and target site duplication. Evaluation of the redundancy of the MITE in nucleotide sequences was 
conducted by BLAST searches using MITE Alex as a query against public databases of $C$. canephora $(56,231)$ Expressed Sequence Tags (EST) (with an $e$-value cut-off of $10 e^{-4}$ ).

2.4. PCR Amplifications. Two primers were designed in conserved regions according to the $46 \mathrm{C} 02 \mathrm{BAC}$ sequence (accession no. EU164537) to check the presence/absence of the MITE Alex-1 at the $g 3$ locus in the different accessions used in this study. The forward primer was designed in the 11th exon of that gene and the reverse primer in the 13th exon (see Supporting Material 1). The primer sequences used were:

G3F: $5^{\prime}$ GTT-TGG-TTG-CTG-GGT-CTC-AT $3^{\prime}$ and G3R: 5' CGA-CAA-GAG-GAA-AGC-CTC-AC 3'. The expected amplicon is $1093 \mathrm{bp}$ long when the MITE Alex-1 is present and $916 \mathrm{bp}$ when it is absent (see supporting Material $1)$.

The PCR conditions were $94^{\circ} \mathrm{C}$ for $1 \mathrm{~min}$. followed by 35 cycles at $94^{\circ} \mathrm{C}$ for $1 \mathrm{~min}, 58^{\circ} \mathrm{C}$ for $30 \mathrm{sec}, 72^{\circ} \mathrm{C}$ for $45 \mathrm{sec}$, and a final elongation period at $72^{\circ} \mathrm{C}$ for $4 \mathrm{~min}$. PCR products were observed by electrophoresis in $1 \%$ agarose gel after staining with ethidium bromide.

2.5. PCR Product Sequencing. In order to check the absence of insertion-as oppose to excision-of a former inserted MITE Alex-1 at the $g 3$ locus, several PCR products from different species were sequenced. After electrophoresis, the bands at 1093 or $916 \mathrm{bp}$ were excised from the gel using a razor blade. DNA was purified using a Quiagen PCR purification kit according to the manufacturer's recommendations and sent for sequencing to Eurofins-MWG (Ebersberg, Germany). Sequences were aligned using ClustalW software.

\section{Results}

3.1. Identification of Alex, a Novel MITE Family, in C. canephora. A BAC clone at the CcEIN4 locus in C. canephora (BAC clone 46C02, accession no. EU164537) was recently sequenced [17]. This represented the first complete BAC clone ever sequenced in the Coffea genus. The typical structural features of a Miniature Invertedrepeat Transposable Element (MITE) were detected in the 12 th intron of the $g 3$ gene, encoding a putative protein (nucleotides 12468...12645 of the BAC clone). This element, named Alex-1, was flanked by the 3 bp direct repeat AGT, generated upon the insertion of the element (Target Site Duplication, TIR) and had 18 bp Terminal Inverted Repeats (TIR) at both ends of the element. The small sequence size (178 bp), rich A/T composition (73.4\%), and the ability to form secondary structures characterized Alex-1 (Figure 1).

To further characterize this element, the nucleotide sequence of Alex-1 was compared with the public nucleotide sequences of C. canephora comprising 56,231 Expressed Sequence Tags. BLAST searches produced 42 significant hits, suggesting that Alex-1 belongs to a large family of MITE elements frequently associated with transcribed sequences. A BLASTN search performed on nonredundant (nr) public libraries did not produce any significant hit (length, percentage, identity, and $e$-value), except in Coffea genomic sequences.

3.2. Analysis of Genomic Polymorphism Associated with the MITE Alex-1 at the g3 Locus. Table 1(a) shows the presence or absence of the Alex-1 MITE at the g3 locus in the different plants analyzed. Within-species polymorphism in C. canephora is presented in Table $1(\mathrm{~b})$ (see Supporting Material 3).

The majority of the species (18/28) displayed total absence of the MITE at the $\mathrm{g} 3$ locus, whereas $7 / 28$ were homozygous for its presence and $3 / 28$ displayed heterogeneous patterns. Only C. liberica var. devewrei and C. canephora displayed all three genotypes, homozygous $+/+$, homozygous $-/-$, and heterozygous $+/-$.

Two species or taxons showed a majority of homozygous genotypes, $+/+$ (C. sp N'Koumbala) or $-/-($ C. humilis), few heterozygotes + /-, but not the reciprocal homozygous, -I(C. sp N'Koumbala) or $+/+$ (C. humilis).

At the sequence level, a closer look at the locus of insertion for the presence of Alex-1 in both positive and negative plants showed that only one of the negative individuals, C. anthonyi, displayed the remnant of a TSD (Target Site Duplication) sequence that indicates the former presence of a MITE and thus its transposition (Figure 2). This process of excision was precise since the whole element was removed from the site of insertion, and no large deletion occurred in the flanking regions.

Interestingly, in the African species, no link was found between the geographical origin of the species and the presence of the Alex-1 MITE. Indeed, Alex-1 was present in East, West and Central African species (Mozambicoffea and Eucoffea, resp.). However, all the Mascarocoffea species originating from islands in the Indian Ocean (Madagascar, Mauritius, and Comoros) lacked the MITE at the $g 3$ locus. Most Coffea species appear to be homozygous since only seven plants out of 129 were heterozygous.

The situation of C. canephora is particularly interesting since the only diversity group E, originating from the Congo/Cameroon region, contains homozygous - Igenotypes (Table 1(b) and see Supporting Material 4). The hetero-zygotes detected in groups $\mathrm{D}, \mathrm{A}$, and $\mathrm{C}$ were previously identified as being intergroup hybrids all with a group E genotype in their pedigree [19]. Similarly, heterozygotes in group E turned out to be hybrids between group $\mathrm{D}$ and group E genitors [19]. The homozygous individual in group E was collected in RCI (Ivory Coast), far from the place of origin of that diversity group, which is in the Congo/Cameroon region. This particular plant was certainly introduced into RCI for improvement purposes quite a long time ago and has certainly undergone several crosses and backcrosses leading to an introgressed form bearing the inserted locus on both homologous chromosomes (+/+) (Accession 319, see Supporting Material 2 and 4). The diversity group E thus appears to be the only one among the $C$. canephora groups to be characterized by the absence of Alex-1 at the $g 3$ locus. 
TABLE 1

(a) Insertion polymorphism of the Alex-1 MITE at the g3 locus among a representative set of Coffea species and a close relative Psilanthus ebracteolatus.

\begin{tabular}{|c|c|c|c|c|c|}
\hline Species analyzed & No. of individuals & $+/+$ & $-1-$ & $+1-$ & Origin \\
\hline C. arabica & 3 & 3 & 0 & 0 & E. Africa, Ethiopia \\
\hline C. eugenioides & 9 & 9 & 0 & 0 & E. Africa Kenya \\
\hline C. pseudozanguebariae & 11 & 0 & 11 & 0 & E. Africa Kenya \\
\hline C. racemosa & 11 & 0 & 11 & 0 & E. Africa Tanzania \\
\hline C. liberica var liberica & 10 & 0 & 10 & 0 & W. Africa RCI \\
\hline C. stenophylla & 10 & 0 & 10 & 0 & W. Africa RCI \\
\hline C. humilis & 10 & 0 & 8 & 2 & W. Africa RCI \\
\hline C. canephora & 71 & 53 & 8 & 10 & W. and C. Africa \\
\hline C. congensis & 5 & 5 & 0 & 0 & C. Africa RCA \\
\hline C. liberica var. dewevrei & 10 & 4 & 3 & 3 & C. Africa RCA \\
\hline C. liberica var. koto & 3 & 3 & 0 & 0 & C. Africa Cameroon \\
\hline C. brevipes & 10 & 10 & 0 & 0 & C. Africa Cameroon \\
\hline C. heterocalyx & 1 & 0 & 1 & 0 & C. Africa Cameroon \\
\hline C. anthonyi & 7 & 0 & 7 & 0 & C. Africa Cameroon \\
\hline C. $s p$ N'Koumbala & 10 & 8 & 0 & 2 & C. Africa Cameroon \\
\hline C. sp Mayombé & 3 & 3 & 0 & 0 & C. Africa Congo R. \\
\hline C. kapakata & 2 & 2 & 0 & 0 & C. Africa Angola \\
\hline C. myrtifolia & 3 & 0 & 3 & 0 & Mauritius \\
\hline C. resinosa & 1 & 0 & 1 & 0 & Madagascar \\
\hline C. tsirananae & 1 & 0 & 1 & 0 & Madagascar \\
\hline C. lancifolia & 1 & 0 & 1 & 0 & Madagascar \\
\hline C. perrieri & 1 & 0 & 1 & 0 & Madagascar \\
\hline C. sakarahae & 1 & 0 & 1 & 0 & Madagascar \\
\hline C. millotii & 1 & 0 & 1 & 0 & Madagascar \\
\hline C. dolichophyla & 1 & 0 & 1 & 0 & Madagascar \\
\hline C. heimii & 1 & 0 & 1 & 0 & Madagascar \\
\hline C. bertrandii & 1 & 0 & 1 & 0 & Madagascar \\
\hline C. humblotiana & 1 & 0 & 1 & 0 & Comoros \\
\hline P. ebracteolatus & 1 & 0 & 1 & 0 & W. Africa RCI \\
\hline Total & 200 & 100 & 83 & 17 & \\
\hline
\end{tabular}

E. Africa: East Africa; W. Africa: West Africa; C. Africa: Central Africa.

RCI: République de Côte d’Ivoire (Ivory Coast); RCA: République Centre Africaine (Central African Republic).

(b) Insertion polymorphism of the Alex-1 MITE at the 83 locus among a representative set of the diversity groups of the Coffea canephora species as defined by Gomez et al. [19].

\begin{tabular}{lccccc}
\hline Diversity group & No. of individuals & $+/+$ & $-/-$ & $+/-$ & Origin \\
\hline D & 29 & 28 & 0 & $1^{*}(\mathrm{DEA})$ & Guinea/RCI \\
$\mathrm{A}$ & 2 & 1 & 0 & $1^{*}(\mathrm{AE})$ & Cameroon/Congo \\
$\mathrm{B}$ & 3 & 3 & 0 & 0 & $\mathrm{RCA}$ \\
$\mathrm{C}$ & 9 & 8 & 0 & $1^{*}(\mathrm{CE})$ & Cameroon/Congo/RCA \\
$\mathrm{E}$ & 16 & 1 & 8 & $7^{*}(\mathrm{DE})$ & Congo/Cameroon/RCA \\
O & 12 & 12 & 0 & 0 & Uganda \\
\hline Total & 71 & 53 & 8 & 10 & \\
\hline
\end{tabular}

$+/+\&-/-$ : Homozygote for presence and absence, respectively; +/-: Heterozygote. ${ }^{*}$ : intraspecific hybrids. 

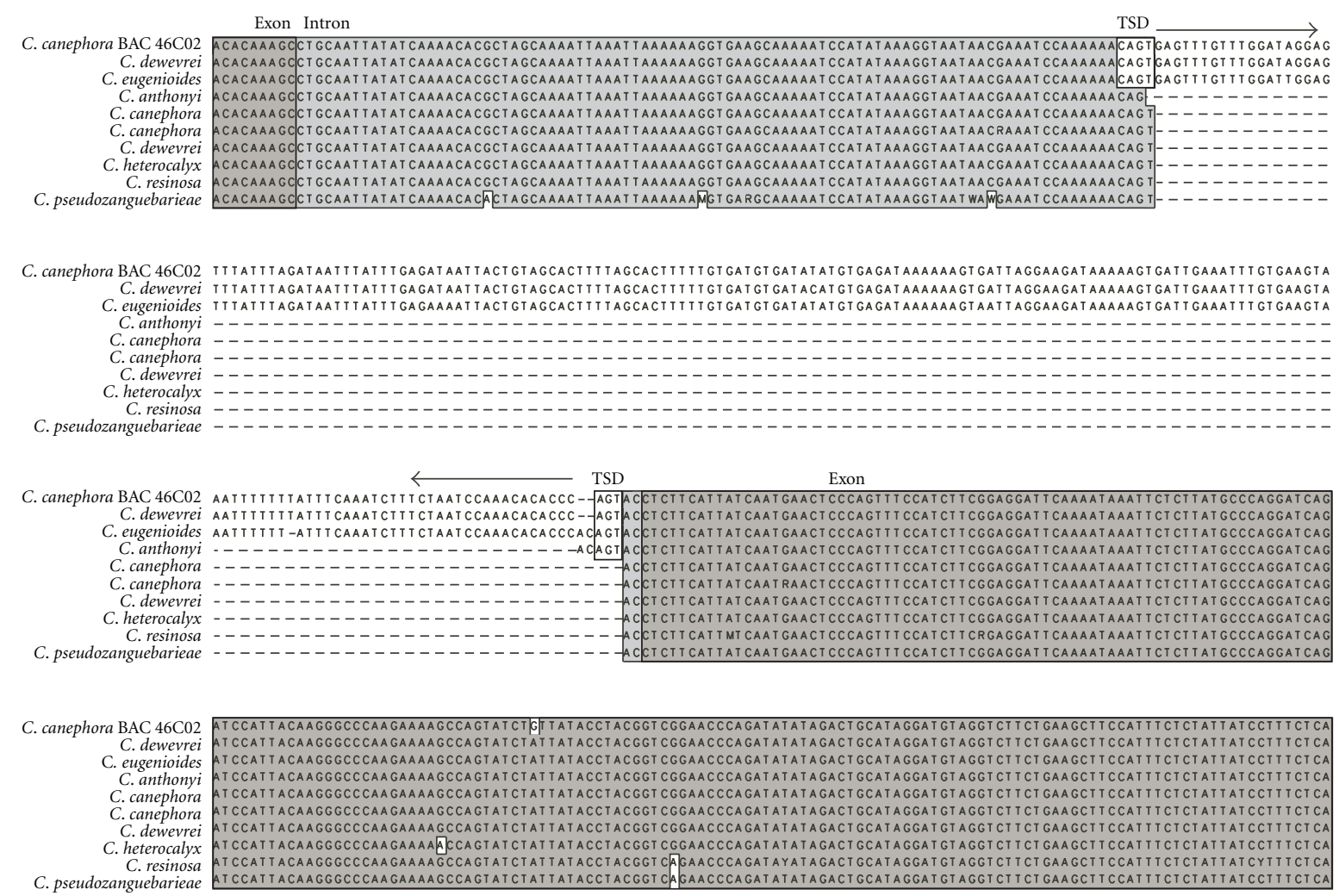

FIGURE 2: Sequence alignment of Alex-1 insertion site in different Coffea species. C. anthonyi is the only negative sequence displaying the presence of a Target Site Duplication (TSD) (empty boxes in the figure). Black arrows indicate the presence of the Terminal Inverted Repeats (TIRs).

Analysis of the sequences in seven negative genotypes in the E diversity group and in negative genotypes in other diversity groups revealed no remnant TSD sequences, suggesting that the absence of Alex-1 at the 83 locus is more likely due to a lack of insertion than to postdifferentiation excision (Figure 3).

The closest relative to the genus Coffea, a plant from the Psilanthus genus, also lacked the insertion of the Alex-1 MITE at the $g 3$ locus.

\section{Discussion}

In this paper we describe the first MITE characterized in the genome of a Coffea species that has no homologs in other published sequences, it appears thus as specific to the Coffea genus. We studied the insertion polymorphism of this MITE at the $g 3$ locus. The distribution pattern of Alex-1 at this locus in the Coffea and Psilanthus species strongly suggests that insertion occurred early in relation with the evolution of these genera, although certainly after the divergence between Coffea and Psilanthus as Alex-1 was not found in Psilanthus. However, not enough Psilanthus species or genotypes were analyzed to confirm this hypothesis.

Because the distribution of the MITE Alex-1 does not corroborate previous phylogenetic studies [13] and was found in Coffea species independently of their eco-geographical distribution (Figure 4), its insertion at the $g 3$ locus most probably occurred before the spread of the genus in Africa but probably after the colonization of Madagascar and the other islands in the Indian Ocean by one or several ancestral Coffea species if we consider the hypothesis that the genus originated in the African continent.

Its insertion certainly occurred before the formation of the $C$. arabica species, which is the only allotetraploid in the Coffea genus, originating from Southern Ethiopia and most probably resulting from a cross between $C$. eugenioides and C. canephora [23] both being only or mostly homozygous + l+.

When the insertion of a transposable element occurs, it is always in heterozygous form. The probability that the same TE is inserted at the same locus, at exactly the same spot on both homologous chromosomes, is almost nil. If the insertion does not modify a gene function leading to an advantage or disadvantage in terms of selection, its mainten-ance in the genome responds to a neutral model and may be conserved or eliminated in the following generations. In the present case, as no link was found between the presence or absence of Alex-1 at the $g 3$ locus and the habitat type of the species, the neutral situation probably applies. It is still not clear why species then became preferentially fixed for the pre-sence or absence of the TE, if this was not merely random.

Four species displayed the presence of heterozygous genotypes and only two (C. canephora and C. liberica var dewevrei) showed the three possible patterns (Table 1(a)), 

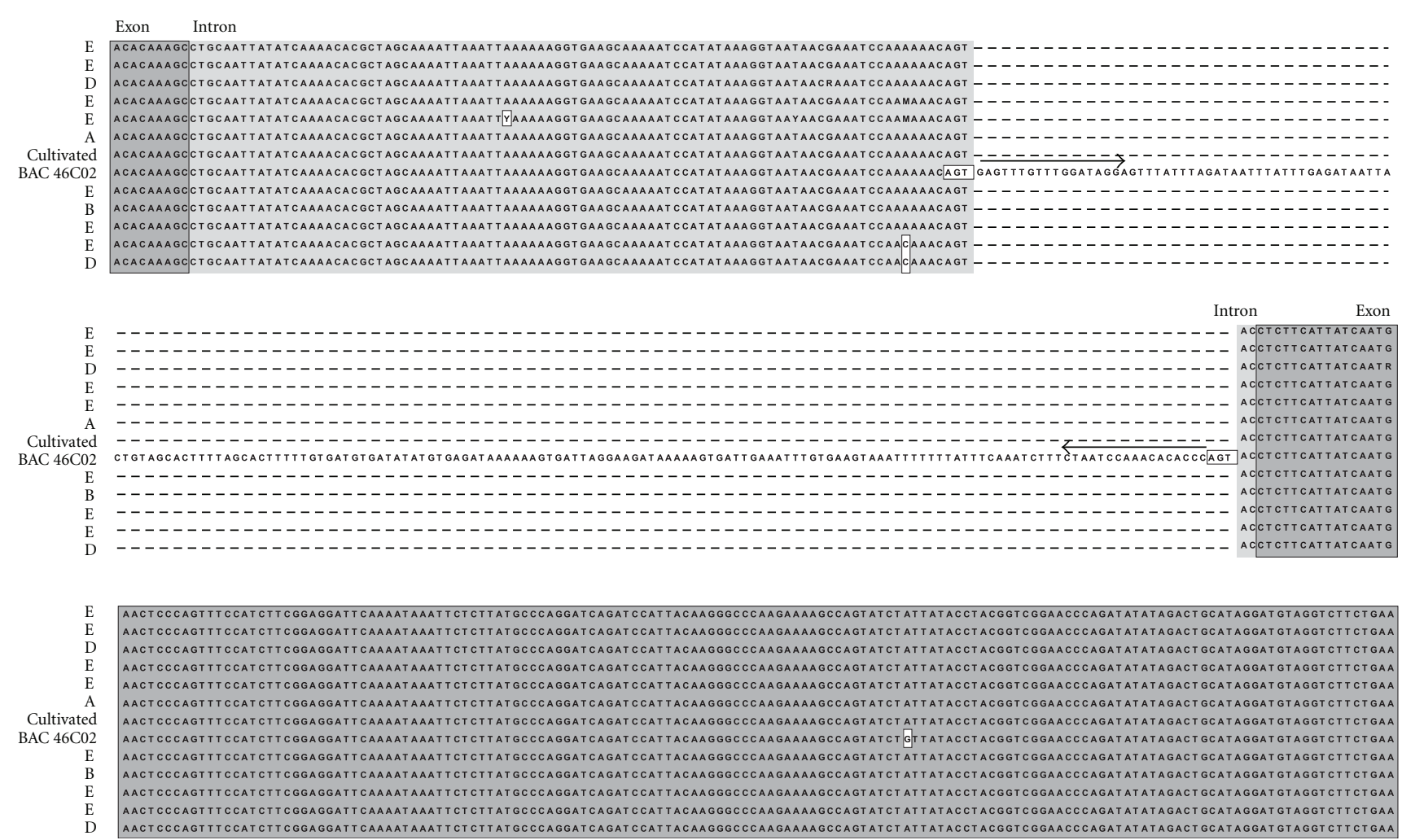

FIgure 3: Sequence alignment of $C$. canephora accessions displaying the absence of Alex-1 compared with BAC 46C02 sequence. The absence of a TSD indicates that the MITE was not excised from this site and that insertion never occurred. The accessions sequences were 739, 504, $738,665,663,730,321,604,345,651$, and 725 for wild accessions belonging to A, B, D, and E genetic diversity groups, and to the cultivated BD55 accession from Cameroon. Empty boxes: TSD. Black arrows: TIRs.

homozygous $(+/+$ and $-/-)$ and heterozygous $(+/-)$. It is very likely that because of the size of the sample, all possible situations have not yet been identified in all the species. It is also possible that some fixation and/or divergence events are actually still underway. The most intriguing example is C. liberica var dewevrei, which displays the three genotypes (homozygous and heterozygous), while C. liberica var liberica is fixed for the absence of Alex-1 (homozygotes -/-) and C. liberica var koto is fixed for the presence of Alex-1 (homozygotes $+/+$ ). C. liberica var dewevrei may still be in the fixation process but this could take quite a long time, as the three genotypes are encountered with equal frequency.

In the cases of C. sp N'Koumbala and C. humilis, no -/or $+/+$ homozygotes were identified (Table 1(a)), which does not mean that these types of homozygotes do not exist but simply that they were not present in the sample we analyzed. The presence of heterozygotes $(+/-)$ can result from an allelic equilibrium with a low frequency of positive alleles in C. sp N'Koumbala and of a negative allele in C. humilis, but in such a situation, the reciprocal homozygote would also be expected to be present, and this was not the case in our sample. Another possible explanation for this low allelic frequency is that interspecific crosses, even if very rare, may happen throughout the Cameroon/Congo region and in RCI, which are hot spots of diversification and secondary centers of speciation for Coffea species [24].
The C. canephora group $\mathrm{E}$ insertion pattern suggests possible interspecific hybridization and gene flow. Indeed, it is the only group in this species that lacked Alex-1 at the g3 locus. The absence of TSD in the sequenced amplicons (Figure 3) indicates that Alex-1 has never been inserted at that locus, and consequently, that its absence is not the result of transposition to another site. All the other genotypes, whatever diversity group they belong to, are $+/+$ homozygotes, it is thus highly likely that the common ancestor of $C$. canephora underwent the insertion of Alex1 at the $g 3$ locus and then evolved towards the fixation of the insertion (+/+ pattern). Group E, and certainly other unidentified genomic sequences, could then result from an introgression following a cross with a neighboring (sympatric) species and backcrosses to recover C. canephora properties.

Interestingly, under this hypothesis of introgression, $C$. $s p$ N'Koumbala is a possible candidate to be the provider of the absence of insertion if an allelic equilibrium remains in this species. Indeed, this taxon grows in the same region as plants from diversity group E, but additional comparative sequencing of the $g 3$ locus, including flanking regions, is necessary to confirm or reject the hypothesis.

It is also possible that this particular group derives from a sister plant to the plant that integrated the MITE, the latter 


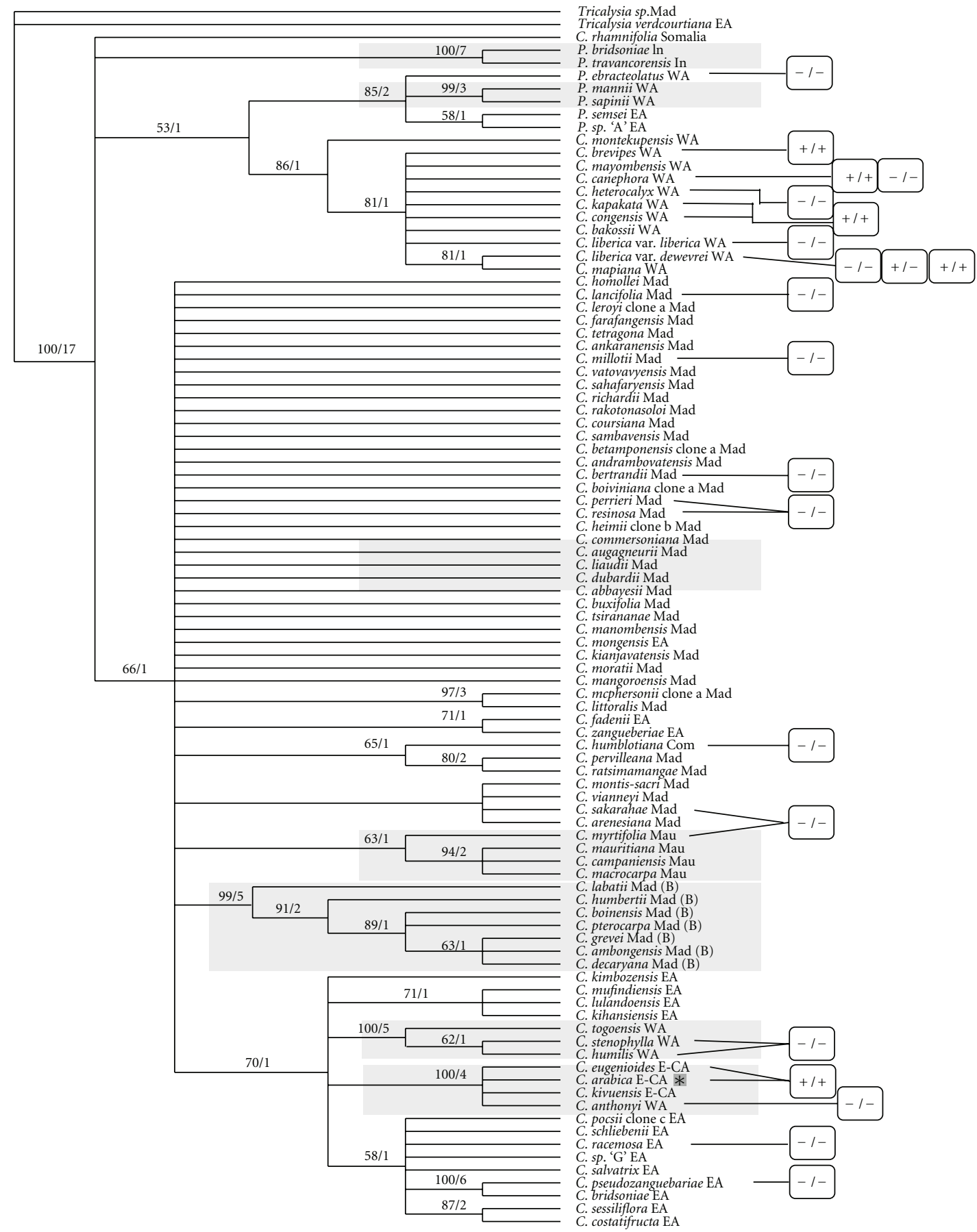

FIGURE 4: Insertion polymorphism of the MITE Alex-1 superimposed to a phylogenetic tree established by Maurin et al. [13]. Except for the species from the Indian Ocean islands, which are homozygous -/-, all possible allelic associations are found on the African continent despite the geographical region, West or East. *: C. arabica is the sole tetraploid Coffea species. WA: West and Central Africa, Mad: Madagascar, Com: Comoros, Mau: Mauritius, EA: East Africa. All the species analyzed in the present study are not represented on the tree and reciprocally.

resulted in the full $C$. canephora lineage except for group E. In this case, group $\mathrm{E}$ may derive from a population that lived in sympatry with C. canephora, from which it has never completely genetically separated due to cross hybridization. If this is the case, the genome region that contains the Alex-1 MITE was preserved from recombination, which should have led to $+/-$ and $+/+$ genotypes. However, genotypes that are found in artificial intraspecific hybrids make this hypothesis unlikely.

\section{Conclusion}

The insertion pattern of the Alex-1 MITE at the $g 3$ locus in Coffea species indicates an original path of speciesdifferen- 
tiation including gene flows between ancestral forms that happened before the present. Recent collecting missions very occasionally identified natural interspecific hybrids or sympatric populations of Coffea species. However, it is known that such events can happen in the wild (C. arabica being the best example), or in displaced populations in functioning or abandoned coffee plantations [25]. Changing environmental conditions and habitat modification could certainly have led to cohabitation of two or more species in limited areas where their specific phenology was disturbed, thus allowing cross pollination. Subsequent environmental changes could have led to the expansion of favorable habitats, resulting in the isolation of the newly formed species.

MITEs thus appear to be a powerful tool to analyze these speciation events and to trace the phylogenetic relationships between species and if the number of specific insertion sites is sufficient to enable the establishment of an event chronology $[8]$.

\section{Acknowledgments}

The authors thanks go to P. Musoli from NARO/COREC (Uganda) and T. Leroy from CIRAD (France) for kindly providing DNA from Ugandan C. canephora.

\section{References}

[1] S.-J. Kwon, K. L. Ju, S. W. Hong, Y. J. Park, K. L. McNally, and N. S. Kim, "Genetic diversity and phylogenetic relationship in AA Oryza species as revealed by Rim2/Hipa CACTA transposon display," Genes and Genetic Systems, vol. 81, no. 2, pp. 93-101, 2006.

[2] K. Takagi, H. Nagano, Y. Kishima, and Y. Sano, "MITE-transposon display efficiently detects polymorphisms among the Oryza AA-genome species," Breeding Science, vol. 53, no. 2, pp. 125-132, 2003.

[3] S. J. Wheelan, L. Z. Scheifele, F. Martinez-Murillo, R. A. Irizarry, and J. D. Boeke, "Transposon insertion site profiling chip (TIP-chip)," Proceedings of the National Academy of Sciences of the United States of America, vol. 103, no. 47, pp. 17632-17637, 2006.

[4] Z. J. Xu and W. Ramakrishna, "Retrotransposon insertion polymorphisms in six rice genes and their evolutionary history," Gene, vol. 412, no. 1-2, pp. 50-58, 2008.

[5] M. Calonje et al., "Non-coding nuclear DNA markers in phylogenetic reconstruction," Plant Systematics and Evolution, vol. 282, no. 3-4, pp. 257-280, 2009.

[6] J. M. Casacuberta and N. Santiago, "Plant LTR-retrotransposons and MITEs: control of transposition and impact on the evolution of plant genes and genomes," Gene, vol. 311, no. 1-2, pp. 1-11, 2003.

[7] D. Grzebelus et al., "Population dynamics of miniature inverted-repeat transposable elements (MITEs) in Medicago truncatula," Gene, vol. 448, no. 2, pp. 214-220, 2009.

[8] R. J. Mason-Gamer, "Multiple homoplasious insertions and deletions of a Triticeae (Poaceae) DNA transposon: a phylogenetic perspective," BMC Evolutionary Biology, vol. 7, article 92, 2007.

[9] C. Feschotte, N. Jiang, and S. R. Wessler, "Plant transposable elements: where genetics meets genomics," Nature Reviews Genetics, vol. 3, no. 5, pp. 329-341, 2002.
[10] A. M. Casa, S. E. Mitchell, O. S. Smith, J. C. Register, S. R. Wessler, and S. Kresovich, "Evaluation of $\mathrm{Hbr}$ (MITE) markers for assessment of genetic relationships among maize (Zea mays L.) inbred lines," Theoretical and Applied Genetics, vol. 104, no. 1, pp. 104-110, 2002.

[11] S. R. Wessler, T. E. Bureau, and S. E. White, "LTR-retrotransposons and MITEs: important players in the evolution of plant genomes," Current Opinion in Genetics and Development, vol. 5, no. 6, pp. 814-821, 1995.

[12] A. Benjak, S. Boue, A. Forneck, and J. M. Casacuberta, "Recent amplification and impact of MITEs on the genome of grapevine (Vitis vinifera L.)," Genome Biology and Evolution, vol. 1, pp. 75-84, 2009.

[13] O. Maurin, A. P. Davis, M. Chester, E. F. Mvungi, Y. Jaufeerally-Fakim, and M. F. Fay, "Towards a phylogeny for Coffea (Rubiaceae): identifying well-supported lineages based on nuclear and plastid DNA sequences," Annals of Botany, vol. 100, no. 7, pp. 1565-1583, 2007.

[14] R. Waugh, K. McLean, A. J. Flavell et al., "Genetic distribution of Bare-1-like retrotransposable elements in the barley genome revealed by sequence-specific amplification polymorphisms (S-SAP)," Molecular and General Genetics, vol. 253, no. 6, pp. 687-694, 1997.

[15] R. Kalendar, T. Grob, M. Regina, A. Suoniemi, and A. Schulman, "IRAP and REMAP: two new retrotransposon-based DNA fingerprinting techniques," Theoretical and Applied Genetics, vol. 98, no. 5, pp. 704-711, 1999.

[16] A. J. Flavell, M. R. Knox, S. R. Pearce, and T. H. N. Ellis, "Retrotransposon-based insertion polymorphisms (RBIP) for high throughput marker analysis," Plant Journal, vol. 16, no. 5, pp. 643-650, 1998.

[17] R. Guyot et al., "Microcollinearity in an ethylene receptor coding gene region of the Coffea canephora genome is extensively conserved with Vitis vinifera and other distant dicotyledonous sequenced genomes," BMC Plant Biology, vol. 9, no. 1, article 22, 2009.

[18] P. Cubry et al., "Diversity in coffee assessed with SSR markers: structure of the genus Coffea and perspectives for breeding," Genome, vol. 51, no. 1, pp. 50-63, 2008.

[19] C. Gomez, S. Dussert, P. Hamon, S. Hamon, A. de Kochko, and V. Poncet, "Current genetic differentiation of Coffea canephora pierre ex a. Froehn in the guineo-Congolian african zone: cumulative impact of ancient climatic changes and recent human activities," BMC Evolutionary Biology, vol. 9, no. 1, article 167, 2009.

[20] P. Musoli, P. Cubry, P. Aluka et al., "Genetic differentiation of wild and cultivated populations: diversity of Coffea canephora Pierre in Uganda," Genome, vol. 52, no. 7, pp. 634-646, 2009.

[21] P. Hamon et al., "Two novel Ty1-copia retrotransposons isolated from coffee trees can effectively reveal evolutionary relationships in the Coffea genus (Rubiaceae)," Molecular Genetics and Genomics, vol. 285, no. 6, pp. 447-460, 2011.

[22] E. L. L. Sonnhammer and R. Durbin, "A dot-matrix program with dynamic threshold control suited for genomic DNA and protein sequence analysis," Gene, vol. 167, no. 1-2, pp. GC1GC10, 1995.

[23] P. Hamon, S. Siljak-Yakovlev, S. Srisuwan et al., "Physical mapping of rDNA and heterochromatin in chromosomes of 16 Coffea species: a revised view of species differentiation," Chromosome Research, vol. 17, no. 3, pp. 291-304, 2009.

[24] A. P. Davis, R. Govaerts, D. M. Bridson, and P. Stoffelen, "An annotated taxonomic conspectus of the genus Coffea (Rubiaceae)," Botanical Journal of the Linnean Society, vol. 152, no. 4, pp. 465-512, 2006. 
[25] C. Gomez et al., "Favourable habitats for Coffea inter-specific hybridization in central New Caledonia: combined genetic and spatial analyses," Journal of Applied Ecology, vol. 47, no. 1, pp. 85-95, 2010. 

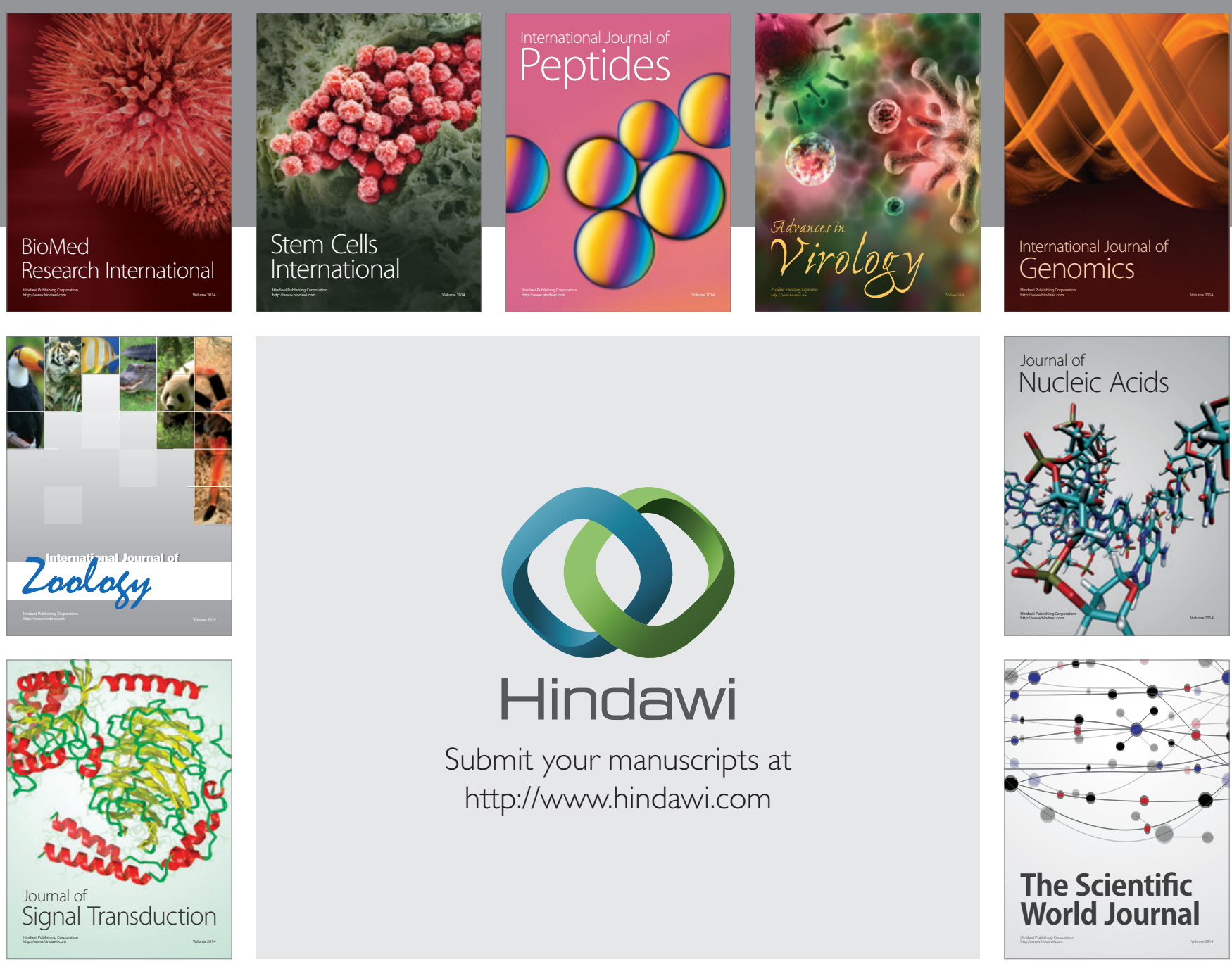

Submit your manuscripts at

http://www.hindawi.com
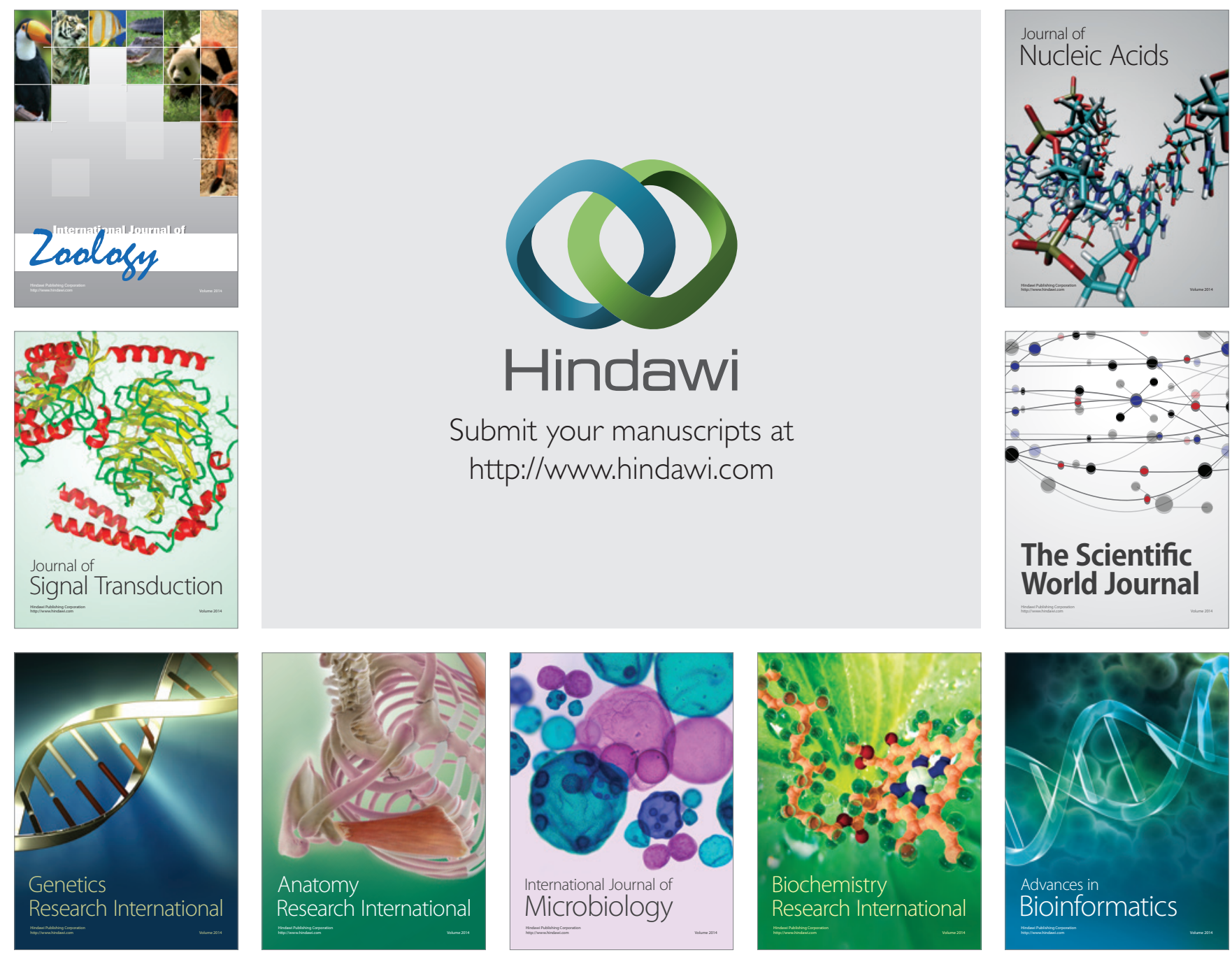

The Scientific World Journal
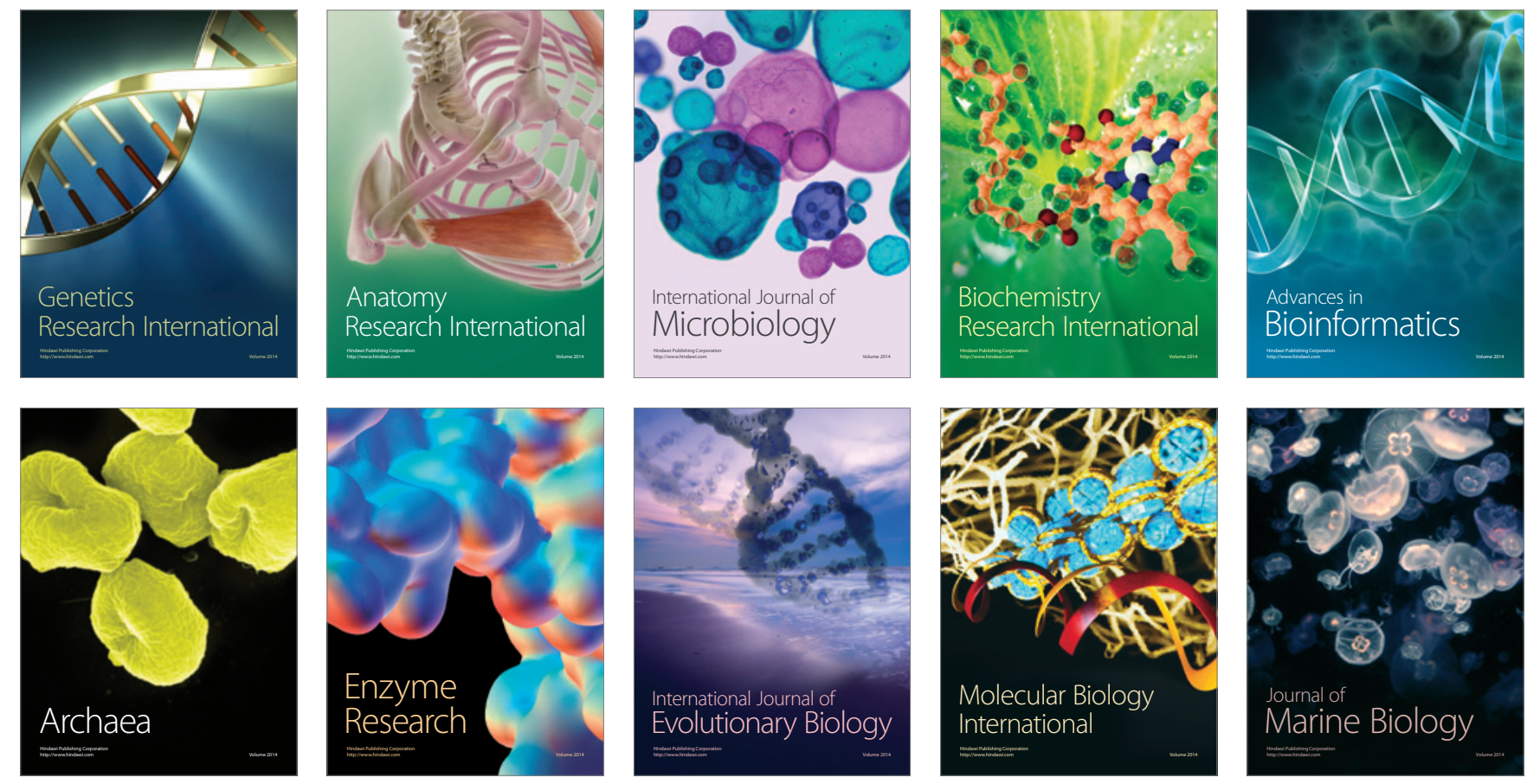